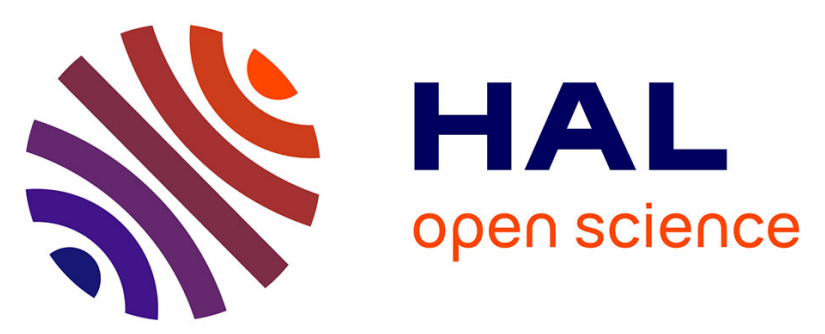

\title{
Pathways control in modification of solid surfaces induced by temporarily separated femtosecond laser pulses
}

L. Museur, A. Manousaki, D. Anglos, G.D. D Tsibidis, A. Kanaev

\section{- To cite this version:}

L. Museur, A. Manousaki, D. Anglos, G.D. D Tsibidis, A. Kanaev. Pathways control in modification of solid surfaces induced by temporarily separated femtosecond laser pulses. Applied Surface Science, 2021, 566, pp.150611. 10.1016/j.apsusc.2021.150611 . hal-03412155

\section{HAL Id: hal-03412155 \\ https://hal.science/hal-03412155}

Submitted on 2 Nov 2021

HAL is a multi-disciplinary open access archive for the deposit and dissemination of scientific research documents, whether they are published or not. The documents may come from teaching and research institutions in France or abroad, or from public or private research centers.
L'archive ouverte pluridisciplinaire HAL, est destinée au dépôt et à la diffusion de documents scientifiques de niveau recherche, publiés ou non, émanant des établissements d'enseignement et de recherche français ou étrangers, des laboratoires publics ou privés. 


\title{
Pathways control in modification of solid surfaces induced by temporarily separated femtosecond laser pulses
}

\author{
L. Museur a , A. Manousaki b, D. Anglos ${ }^{\text {b }}$, G. D. Tsibidis ${ }^{\text {b }}$ and A. Kanaev ${ }^{\text {c,* }}$ \\ a Laboratoire de Physique des Lasers (LPL), CNRS, Université Sorbonne Paris Nord, 93430 Villetaneuse, France \\ b Institute of Electronic Structure and Laser (IESL), Foundation for Research and Technology (FORTH), N. Plastira 100, \\ Vassilika Vouton, 70013, Heraklion, Crete, Greece \\ c Laboratoire des Sciences des Procédés et des Matériaux (LSPM), CNRS, Université Sorbonne Paris Nord, 93430 \\ Villetaneuse, France \\ * Corresponding author: andrei.kanaev@lspm.cnrs.fr
}

\begin{abstract}
Reaction control by laser light is a unique method of the reaction dynamics mastery in the molecular chemistry. We

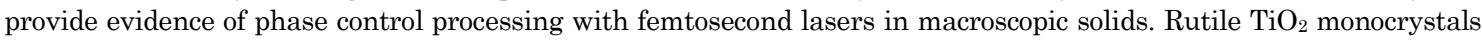
with (001) and (100) surface orientations were irradiated with repetitive pulses of femtosecond KrF laser of variable fluences and a temporal delay between two superimposed linearly polarized beams. The appearance of three types of surface morphology was thoroughly analyzed: low-spatial frequency laser-induced periodic surface structures (LSFL), grooves and unusual featureless flat area (FFA). The interaction of light with the excited surface led to the onset or suppression of the subwavelength LSFL, depending on whether the temporal delay between laser beams is larger or smaller than the critical value of $\sim 6$ ps. By contrast, the suprawavelength grooves and FFA appeared at longer temporal delays. A strong decrease of the grooves onset energy was observed on the (001) oriented crystal after the delay of $\sim 8$ ps; the decrease of onset energy was also observed on the (100) oriented crystal, where FFA appeared instead of grooves. The critical delay is discussed in framework of a phenomenological model describing the energy evolution of excited transient states along the "reaction" coordinate.
\end{abstract}

Keywords: LIPSS; Electronic excitation; TiO $\mathrm{O}_{2}$; Femtosecond UV-laser irradiation.

\section{Introduction}

Femtochemistry is a rapidly developing topic of science since the 1990's, which became an effective method for realisation of the reaction dynamics control of single molecules in a gas phase, molecular beams and in more complex biological systems [1, 2]. In the same time, the control of modifications in solids faces severe restrictions because of the generally much shorter coherence time [3]. Recently, an ultra-high precision subfemtosecond phase monitoring has been employed to validate the approach in condensed matter in several examples of delocalised vibration in solid para-hydrogen, collective phonon motion in bismuth single crystal [4] and electron transfer in CdSe quantum dots [5]. These systems are characterised by a relatively longer picoseconds vibrational dephasing time facilitating the analysis. The very exciting example of the metal-insulator structural phase transition in atomic indium wires on (111) silicon surface via specific phonon modes has been demonstrated only recently [6]. These studies provide some evidence that the interaction can be sensitive to a temporal delay at the femtosecond timescale between two laser beams superimposed on a sample, which affects greatly the quantum states of the system.

The control of the reaction pathways is connected to the transient state evolution along the reaction coordinate, which can lead to the specific bonds breaking, modifying reaction selectivity and final product. This kind of interaction, theoretically considered by Tannor et al. [7], can guide chemical transformations on the ground state potential energy surface of molecular species by intermediary excited states. In the same time, there can be not pronounced similarity between the molecular structure and surface morphology of the crystalline solids [8]. The femtosecond excitation can involve relatively shortlived transient states spanning over the macroscopic volume and creating conditions for the phase control of the energy relaxation process directing the system to a particular surface morphology via the relatively slow melting and re-solidification stages taking place on a longer nanosecond time-scale. In such a case, the reaction pathways might be controlled by the laser fluence, instead of the photon energy assisting molecular transformations in a single molecule. Therefore, the laser fluence and temporal modulation of the absorbed energy are expected to control specific transient states and final reaction state via the density of electronic excitation of solids.

In most representative examples, a weak perturbation in solids was considered. By contrast, in conditions of the strong perturbation, the high density electronic excitation (HDEE) regime can alter the quantum state of a macroscopic crystal. In general, this requires the irradiation of solid surfaces with femtosecond pulses of duration shorter than the electron-phonon relaxation time, of photon energy above the band gap energy of the solid and of fluence above that necessary for producing Mott charge density, which marks the semiconductor-metal transition [9]. Such levels of excitation, when promotes $\sim 10 \%$ electrons to the conduction band, can instantaneously trigger the displacement of cations from their equilibrium positions of the cooled lattice, stabilized by the non-perturbed electron clouds [1013]. The characteristic time of this reorganisation can vary in the 
range between $10^{-12}$ and $10^{-11}$ seconds. Acting on the electronic band structure during this time, may promote the overall macroscopic system to a new transition state with an unusual lattice dynamics, which may guide to a particular final morphology of the irradiated surface.

There is an abundance of experimental and theoretical studies about surface modifications of solids with the ultrashort-pulse laser irradiation. By varying the irradiation conditions of laser fluence $(\mathrm{F})$, number of pulses $(\mathrm{N})$, temporal delay between beams $(\Delta t)$, etc. different laser-induced periodic surface structures (LIPSS) have been obtained and their formation mechanisms have been discussed [14-24]. It has been proposed that very short laser pulses can drive the irradiated matter into a transient "hyperthermal" state far from thermodynamic equilibrium, which is responsible for LIPSS formation [25]. The observation of coherence decay of this intermediate "soft" state with different electronic properties has been suggested for fs-laser ablation by Reif et al. [26] after first indications of an unusual "surface liquid" characterized by an increased absorption of laser photons [27]. An exciting interplay between suppression/amplification of the ablation effectiveness and surface structuring with the delay between laser pulses of the order of several picoseconds has been observed in silicon [28]. Recently, we have analysed the HDEE regime in rutile $\mathrm{TiO}_{2}$ single crystal with two different (100) and (001) surface orientations and reported on their morphological surface maps (MSM) [29], which describe the domains of the appearance of nanoparticle-covered structures (NCS), low-spatial frequency LIPSS (LSFL), grooves and featureless flat area (FFA). The two types of structures, grooves and FFA, respectively appeared on the (001) and (100) oriented surfaces, have been explained by the crystalline anisotropy. The surface patterns in the experimental conditions of HDEE appeared to be connected to the density of electronic excitation (charge carriers density) [30].

In this communication, we analyzed the influence of the temporal delay between two intense femtosecond laser beams impinging the semiconductor surface on MSM and compare energy thresholds of the structures appearance. Rutile $\mathrm{TiO}_{2}$ single crystals with (001) and (100) orientations of the crystalline planes with respect to the surface normal were irradiated. The laser photon energy $(5.0 \mathrm{eV})$ was above the band gap energy of rutile $\mathrm{TiO}_{2}\left(\mathrm{E}_{\mathrm{g}}=3.0 \mathrm{eV}\right.$ [31] $)$ that enables one-photon transitions between the valence and conduction band and, consequently, permits obtaining the highest instantaneous density of the electron-hole plasma (EHP).

\section{Experiment}

The experiments were performed with rutile $\mathrm{TiO}_{2}$ single crystals with (001) and (100) oriented polished surfaces, supplied by Crystal-GmbH, which were shaped in pallets of size 10x10x0.5 $\mathrm{mm}^{3}$. The laser irradiation of crystals was carried out at normal incidence, ambient atmosphere and at room temperature at the Ultraviolet Laser Facility of IESL-FORTH (Heraklion, Greece) with a hybrid distributed feedback dye laser / $\mathrm{KrF}$ excimer system delivering linear polarized pulses of $10 \mathrm{~mJ}$ energy/pulse, $\tau_{L}=450 \mathrm{fs}$ (full width at half maximum) duration at $\lambda_{\mathrm{L}}=248 \mathrm{~nm}$ $\left(\mathrm{h} v_{\mathrm{L}}=5.0 \mathrm{eV}\right)$ and repetition rate $1-10 \mathrm{~Hz}$, which assures effective dissipation of heat between laser pulses and prevents changes of sample temperature during repetitive illumination up to high integral doses, attained with a sequence of $10 \leq \mathrm{N} \leq 10^{3}$ laser pulses. It is noted that the term 'dose' is related to the amount of energy density deposited from the laser on the solid at each single or double pulse.

In pump-pump experiments, the laser beam was split in two beams, as depicted in Fig. 1a, and the temporal delay up to $420 \mathrm{ps}$ (with the precision of $0.5 \mathrm{ps}$ ) was introduced with the Michelson interferometer. The "zero" delay correspondent to the temporal coincidence of the two beams was controlled by the appearance of interference fringes. The energy on the sample was varied by using two reflective attenuators and measured using an energy meter Molectron J04-09 with sensitivity $1.42 \mathrm{~V} / \mathrm{mJ}$. In two-beam irradiation experiment, the energies of the beams were E1 and E2 and the total energy sent to the sample (by one double pulse) was $\mathrm{E}=\mathrm{E} 1+\mathrm{E} 2$. In one-beam irradiation experiment with the same energy E, the delay line (E1) was closed and the beam energy E2 was adjusted to that of the sum of the two beams $\mathrm{E}=\mathrm{E} 1+\mathrm{E} 2$. The laser intensity distribution on sample, after the spatial filtering using a $200 \mathrm{~mm}$ focal distance lens, shown in Fig. 1b, was measured with a camera CM-140 GE-UV from JAI. This picture evidenced a quasi-perfect spatial superposition of the two split and delayed beams within the precision of one pixel $(<5 \mu \mathrm{m})$. The beam-shape measurements, permanently conducted in all experimental series, evidenced a quasi-Gaussian spatial intensity profile on the sample, as shown in the $\mathrm{x}$-axis and $\mathrm{y}$-axis distributions in Fig. 1c. Accordingly, the irradiated spot had a characteristic diameter of $\sim 30 \mu \mathrm{m}$ (measured at 1/e of the maximum intensity), which corresponded to laser fluences on the samples varied between 50 and $2 \cdot 10^{3} \mathrm{~mJ} / \mathrm{cm}^{2}$.
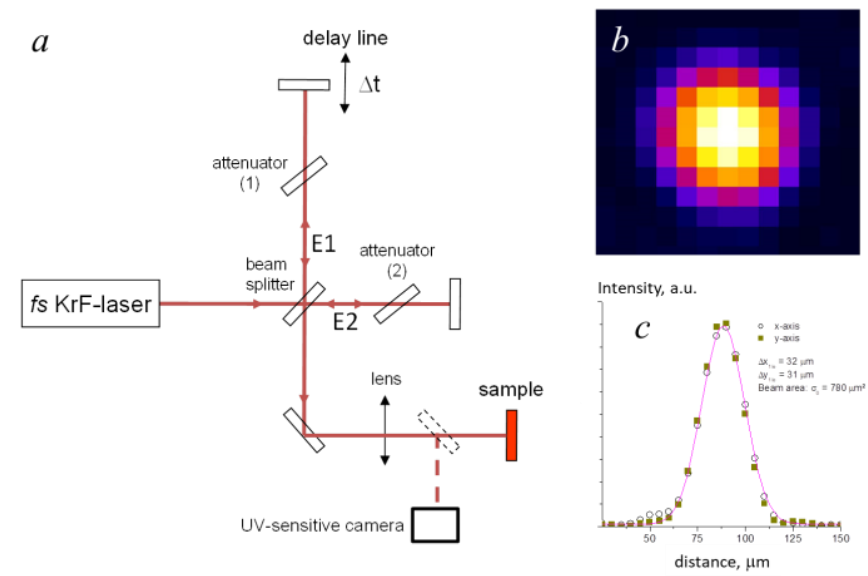

Fig. 1. Experimental schema (a), laser beams superposition (b) and spatial beam shape (c). The size of one pixel is $5 \times 5 \mu \mathrm{m}^{2}(\mathrm{a})$.

The surface morphology after the irradiation was examined by a high-resolution Jeol Scanning Microscope JSM 7000F with a Schottky type field-emission gun (T-FE) operating in a vacuum of $10^{-8} \mathrm{~Pa}$. The spatial resolution was is $1.2 \mathrm{~nm}$ at $30 \mathrm{kV}$ of the maximum accelerating voltage.

\section{Results and discussion}

SEM images of the (001) crystalline surface after one-beam irradiation with $\mathrm{N}=30$, shown in Fig. 2, correspond to the characteristic MSM domains (F, N) described in Ref. [29]. At fluences above a particular value of the threshold $F^{*}=90 \mathrm{~mJ} / \mathrm{cm}^{2}$, LSFL (ripples) were formed as shows image in Fig. 2b. With the 
following increase of the fluence, $1 \mathrm{D}$ pattern transforms to $2 \mathrm{D}$ pattern (Fig. 2c). At very high fluences above $0.6 \mathrm{~J} / \mathrm{cm}^{2}(\mathrm{~N}=100)$, the suprawavelength structure that is termed grooves nucleates and becomes well-developed at $\mathrm{F} \sim 1 \mathrm{~J} / \mathrm{cm}^{2}$. By contrast, at relatively low fluences below $\mathrm{F}^{*}$, NCS (a) were observed (Fig. 2a). The onset of the ripples formation corresponds to a small material sublimation in the irradiated area and is in agreement with the theoretical model of Sipe et al. [32]. The (100) $\mathrm{TiO}_{2}$ surface manifested a similar sequence of the SEM images, except for grooves, which were replaced by FFA.

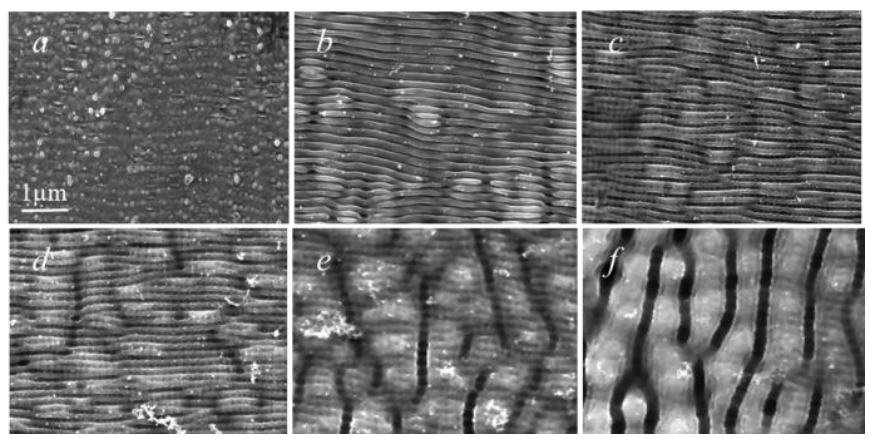

Fig. 2. Surface structuring of rutile $\mathrm{TiO}_{2}(001)$ monocrystal after one-beam irradiation with $\mathrm{KrF}$ laser $(248 \mathrm{~nm}, 450 \mathrm{fs}, \mathrm{N}=100)$ ) fluences are 75 (a), 150 (b), 230 (c), 440 (d), 640 (e) and 960 (f) $\mathrm{mJ} / \mathrm{cm}^{2}$.

An exposition of the semiconductor surface with repetitive pairs of laser pulses with a temporal delay $\Delta t$ between two beams in each pair can significantly modify MSM. A series of SEM images that correspond to the LSFL domain of MSM [29], shown in Fig. 3 , were obtained with the temporal delay of $\Delta t=10$ ps. Fig. $3 a-b$ and $3 \mathrm{~d}-\mathrm{e}$ evidence the initiation of ripples at the periphery of the irradiation spot at (001) and (100) surfaces of rutile $\mathrm{TiO}_{2}$, which is similar to the one-beam experiment (Fig. 2). However, at a longer irradiation with $\mathrm{N}=100$ the central part of the spot was covered with grooves on (001) surface, while FFA appeared on (100) surface, as respectively show images of Fig. 3c and 3f. The appearance of grooves and FFA in the low-energy domain of $\operatorname{MSM}\left(\mathrm{F}=\mathrm{F}_{1}+\mathrm{F}_{2}=300 \mathrm{~mJ} / \mathrm{cm}^{2}\right)$ was totally unexpecting, since these structures are commonly observed at much higher fluencies.
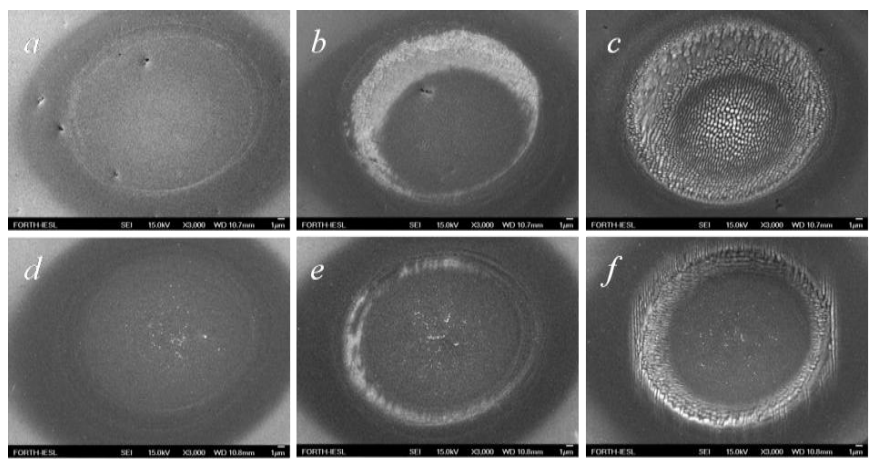

Fig. 3. Surface structuring of rutile $\mathrm{TiO}_{2}(001)(\mathrm{a}-\mathrm{c})$ and (100) (d-f) with repetitive pairs of pulses $\mathrm{N}=5$ (a, d), 20 (b, e), 100 (c, f) of $\mathrm{KrF}$ laser (248 $\mathrm{nm}, 450 \mathrm{fs}$, two-beam with fluences $\mathrm{F}_{1}=100 \mathrm{~mJ} / \mathrm{cm}^{2}$ and $\mathrm{F}_{2}=200 \mathrm{~mJ} / \mathrm{cm}^{2}$ delayed by $10 \mathrm{ps}$ ).

The difference between the groove formation in one-beam and two-beam experiments is further explored with results shown in Fig. 4. In agreement with the commonly accepted mechanism, in one-beam experiments grooves were nucleated at the top of ripples as the number of laser pulses $\mathrm{N}$ increased (Fig. 4a-c). In contrast, SEM images in Fig. 4d-e indicate nucleation of grooves in the central area of the spot in absence of any ripples. At large $\mathrm{N}$, however, SEM pictures of crystals in both experiments looked quite similar (Fig. $4 \mathrm{c}$ and f).
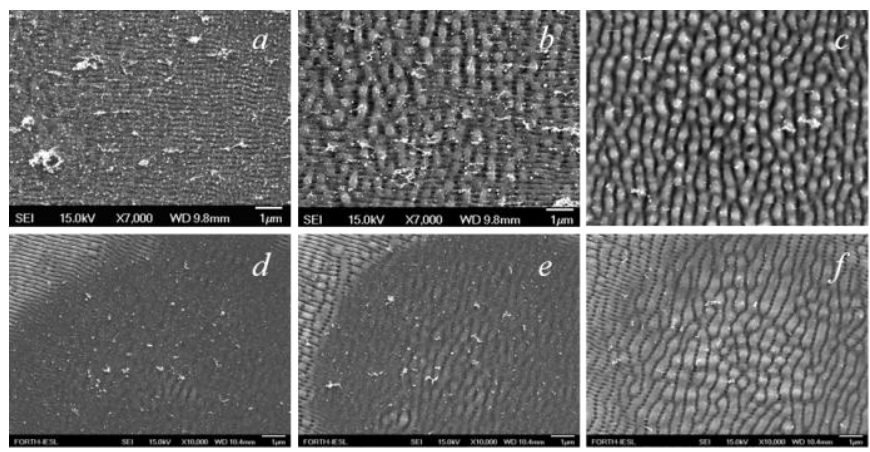

Fig. 4. Surface structuring of rutile $\mathrm{TiO}_{2}(001)$ with $\mathrm{KrF}$ laser $(248 \mathrm{~nm}$, $450 \mathrm{fs}$ ) after one-beam irradiation with $\mathrm{F}=1.1 \mathrm{~J} / \mathrm{cm}^{2}$ and $\mathrm{N}=5(\mathrm{a}), 10$ (b) and 30 -(c) and after two-beam irradiation with $\mathrm{F}_{1}=100 \mathrm{~mJ} / \mathrm{cm}^{2}$ and $\mathrm{F}_{2}=200$ $\mathrm{mJ} / \mathrm{cm}^{2}$ delayed by $10 \mathrm{ps}$ and number of pairs of pulses: $\mathrm{N}=30(\mathrm{~d}), 50(\mathrm{e})$, $100(\mathrm{f})$.

At low fluences but above the threshold of LSFL formation $\left(\mathrm{F}_{1,2}<\mathrm{F}^{*}\right.$ and $\left.\mathrm{F}_{1}+\mathrm{F}_{2} \geq \mathrm{F}^{*}\right)$ and sufficiently long irradiation $(\mathrm{N}=100)$, the surface area covered with ripples Sripples progressively decreased with an increase of the delay time between two laser beams. The dependence of the normalized ratio Sripples/Sbeam on the delay is presented in Fig. 5 for two differently oriented $\mathrm{TiO}_{2}$ crystals. As these results show, the delay time of the ripples disappearance $\tau_{r}$ increased with an increase of the pulse energy, which is in agreement with the hypothesis about energy depletion of the common transient excited state. A critical energy delivered to this state (correspondent to the threshold laser fluence $\mathrm{F}^{*}=90 \mathrm{~mJ} / \mathrm{cm}^{2}$ ) induced the surface morphology evolution towards ripples. The characteristic critical delay time of LSFL disappearance on (100) and (001) oriented surfaces is almost similar and fits the range of $\tau_{\mathrm{r}}=6 \pm 1 \mathrm{ps}$. The overall picture apparently depends on the total energy density absorbed by the crystal to a common transient state, since the disproportioning of the two beams energies did not modify the critical delay time.

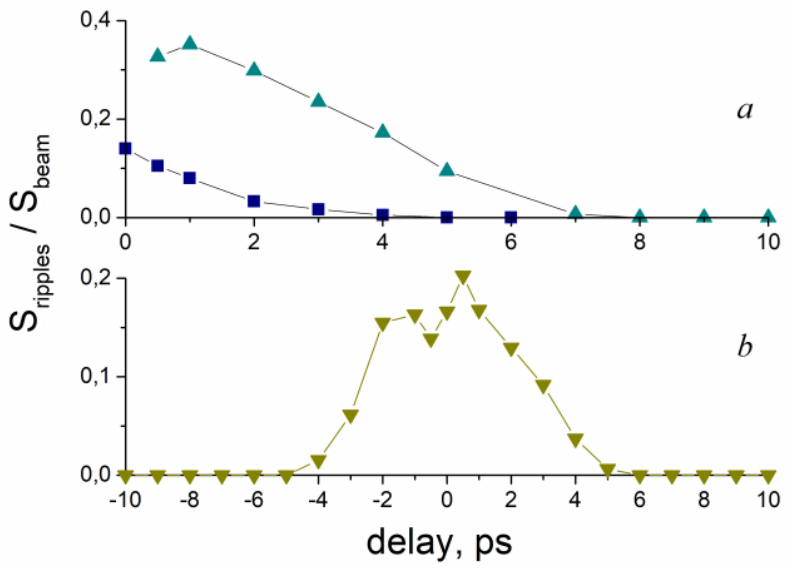

Fig. 5. Relative LSFL-covered area of rutile $\mathrm{TiO}_{2}$ (001) (a) and (100) (b) with two-beam of equal fluences $\mathrm{E}_{1}=\mathrm{E}_{2}=50 \mathrm{~mJ} / \mathrm{cm}^{2}(\mathbf{\Delta}), 75 \mathrm{~mJ} / \mathrm{cm}^{2}(\boldsymbol{\Lambda})$ and two beams of different fluences $\mathrm{E}_{1}=50 \mathrm{~mJ} / \mathrm{cm}^{2}$ and $\mathrm{E}_{2}=70 \mathrm{~mJ} / \mathrm{cm}^{2}(\boldsymbol{\nabla})(\mathrm{KrF}$ laser: $248 \mathrm{~nm}, 450 \mathrm{fs}, \mathrm{N}=100$ ). Sbeam corresponds to the beam area with intensity above $1 / \mathrm{e}$ of the maximum. 
In two-beam experiments with $\mathrm{F}_{1,2} \geq \mathrm{F}^{*}$, each laser pulse separately triggered the formation of LSFL structures (ripples). Furthermore, two more types of surface modification could be observed in this case: grooves on (001) and FFA on (100) oriented rutile $\mathrm{TiO}_{2}$ crystals, which dependence of $\mathrm{S}_{\text {structure/S }} / \mathrm{S}_{\text {beam }}$ on the temporal delay is shown in Fig. 6 for the two crystalline orientations. On the (001) oriented surface, ripples were replaced by grooves: they appeared in the central part of the irradiated spot after a critical delay time $\tau_{\mathrm{g}}$, which shortened from $7 \mathrm{ps}$ to 3 ps with an increase of laser fluence and progressively spanned towards the spot periphery erasing ripples. This supports the previous conclusion of Fig. 4 about different mechanisms of their formation in one-beam and two-beam experiments. We also notice an asymmetry of the structuring area dependence on the delay time, in experiments with disproportioning energies of the two beams (Fig. 6). This point will be discussed below in section concerning possible mechanisms of the structuring.

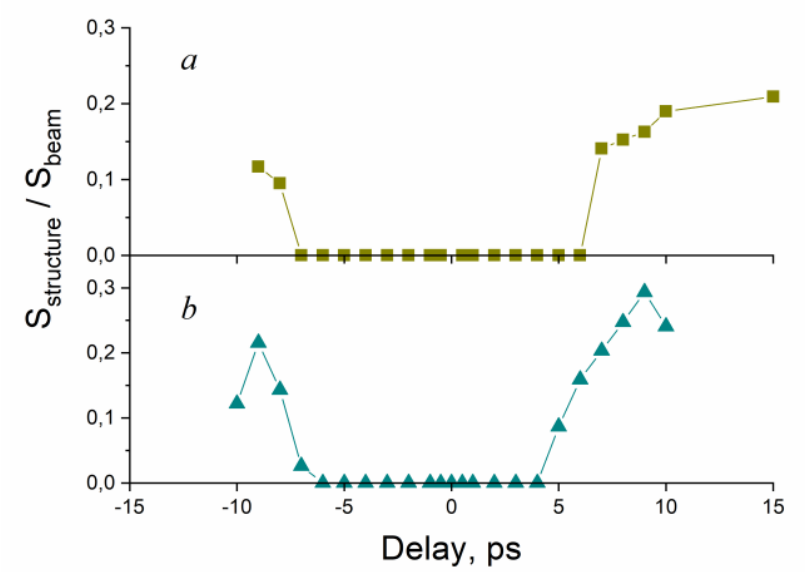

Fig. 6. Relative area of surface structuring of rutile $\mathrm{TiO}_{2}(001)(\mathrm{a})$ and (100) (b) in pump-pump experimens with $\mathrm{F}_{1}=120 \mathrm{~mJ} / \mathrm{cm}^{2}$ and $\mathrm{F}_{2}=240 \mathrm{~mJ} / \mathrm{cm}^{2}$ versus delay between pulses ( $\mathrm{KrF}$ laser: $248 \mathrm{~nm}, 450 \mathrm{fs}, \mathrm{N}=100)$ ): grooves $(\boldsymbol{\square})$ and FFA $(\boldsymbol{\Lambda})$ areas. Sbeam corresponds to the beam area with intensity above $1 / \mathrm{e}$ of the maximum.

The precision of determination of the grooves area on the (100) surface was problematic because of a difficulty with its distinguishing from the area covered with short elongated precursor relief particles, which appeared at the grooves nucleation stage. In contrast, because the net border between structured and flat areas (see tilt SEM images in Fig. 7), (100) oriented crystal enabled precise measurements of the structuring area evolution with temporal delay. The data analysis showed that FFA appeared in the same range of laser fluencies and time delays, as grooves on the (001) surface. Furthermore, SEM images indicated that FFA replaced ripples in the central part of the irradiated spot and, similarly to grooves on the (001) oriented surface, was formed after the critical time delay of $\Delta t \approx \tau_{\mathrm{g}}$ (Fig. 6) between two laser beams. Based on these observations, a general conclusion can be drawn about similar mechanisms responsible for the FFA and grooves formation. The critical delay time $\tau_{\mathrm{g}}$ is apparently due to a transient state (different from that leading to the formation of ripples) excited by the first laser beam, which feeds with energy from the delayed beam.

Complementary to these measurements, Fig. 8 evidences that the critical delay required for the grooves / FFA formation shortens with an increase of the pulse energy. Indeed, fluence of
$350 \mathrm{~mJ} / \mathrm{cm}^{2}$ set up the supra-wavelength structuring at $\Delta \mathrm{t}_{1}=8 \mathrm{ps}$ that disappeared at $\Delta \mathrm{t}_{2}=40 \mathrm{ps}$ by forming submictonic spike-like columns. When increasing fluence up to $600 \mathrm{~mJ} / \mathrm{cm}^{2}$, the timewindow of the grooves onset shortened and shifted to small delays of $3 \leq \Delta t \leq 10 \mathrm{ps}$. This indicates modifications of the transient energetic structure with time.

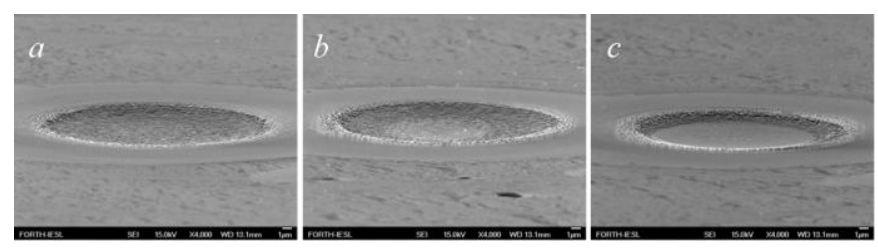

Fig. 7. Tilt SEM images of rutile $\mathrm{TiO}_{2}(100)$ monocrystal irradiated with two laser pulses $(248 \mathrm{~nm}, 450 \mathrm{fs}, \mathrm{N}=100)$ of fluences $\mathrm{F}_{1}=120 \mathrm{~mJ} / \mathrm{cm}^{2}$ and $\mathrm{F}_{2}=240 \mathrm{~mJ} / \mathrm{cm}^{2}$ delayed by $\Delta \mathrm{t}=6 \mathrm{ps}$ (a), $7 \mathrm{ps}$ (b) and $15 \mathrm{ps}$ (c).
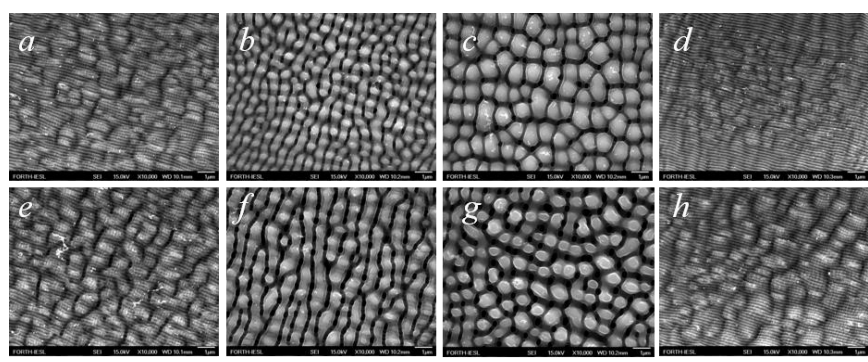

Fig. 8. Surface structuring of rutile $\mathrm{TiO}_{2}(001)$ monocrystal with two laser beams $(248 \mathrm{~nm}, 450 \mathrm{fs}, \mathrm{N}=100)$ of total energy $350 \mathrm{~mJ} / \mathrm{cm}^{2}(\mathrm{a}-\mathrm{d})$ and 600 $\mathrm{mJ} / \mathrm{cm}^{2}$ (e-h) delayed by $\Delta \mathrm{t}=0$ (a, e), $8 \mathrm{ps}(\mathrm{b}), 40 \mathrm{ps}(\mathrm{c}), 3 \mathrm{ps}(\mathrm{f}), 10 \mathrm{ps}(\mathrm{g})$ and $0.1 \mathrm{~s}(\mathrm{~d}, \mathrm{~h})$

The phenomenological schemas of the relevant structuring processes in the pump-pump experiments are depicted in Fig. 9. With respect to the processes which take place and contribute to the topography variation at increasing integral dose (i.e. increase of pulse number), it is noted that hydrodynamical factors play an important role along with electrodynamical effects; more specifically, the latter determine to a great extent the orientation and periodicities of LIPSS while the former lead to the formation of a particular surface topography through transport of a fluid that has been produced following relaxation processes [22]. Fluid transport and resodification of the material determine the surface morphological features that are produced within a few nanoseconds. The timescale of these processes has also been confirmed in previous reports [20, 33-37]. In contrast, the precursor state is activated during the ultrashort double-beam laser irradiation. This precursor state is characterized by the surface energy repartition and survives a time, during which it can be modified by an interaction with the second pulse. We could attribute an energetic structure to this state in terms of X1, $\mathrm{X} 2$ etc. potentials. The mechanism of $\mathrm{X} 1$ state excitation resulting in LSFL consists in the absorption of $1^{\text {st }}$ beam energy, which produces EHP continuum with the under-critical charges number density, which evolves along the reaction coordinate $\mathrm{Q}$ to result in the final NCS morphology. Furthermore, absorption of the $2^{\text {nd }}$ beam energy after the temporal delay $\Delta t$, which defines a partial depletion $\Delta \mathrm{E}_{\text {relaxation }}$ of the initially deposited energy density, can promote the system to X1 state, which trigs LSFL structuring. Considering fluences of both laser beams $\mathrm{F}_{1}, \mathrm{~F}_{2}$ to be smaller than energy density of the ripples onset $\mathrm{E}_{a}$, ripples, the critical time delay $\Delta t=\tau_{r}$, after which ripples cannot be formed, can be defined by 


$$
\mathrm{F}_{1}-\Delta \mathrm{E}_{\text {relaxation }}\left(\tau_{\mathrm{r}}\right)+\mathrm{F}_{2}=\mathrm{E}_{a} \text {, ripples }
$$

The mechanism described by Eq. (1) is schematically depicted in Fig. 9a. In experiments with $\mathrm{F}_{1,2}>\mathrm{F}^{*}$, inhomogeneities of the surface energy distribution produced by the $1^{\text {st }}$ laser beam can be maintained by the $2^{\text {nd }}$ beam under a condition of the process inphase contribution. The characteristic process deactivation time can be estimated as $\tau^{*}=\tau_{\mathrm{r}} \approx 6 \mathrm{ps}$ at laser fluences close to the threshold $\mathrm{F}^{*}$. Beyond this time, the additivity of two contributions is seemingly cancelled suppressing ripples formation, as evidences Fig. 4d. Even when the delayed beam by $\Delta t>\tau^{*}$ is capable producing ripples, its interaction with surface state prepared by the leading beam cancels the formation. Thus, two processes of the LSFL nucleation annihilate in these conditions.

The case of the grooves formation in the conditions of $F_{1,2}>F^{*}$ is more complex. On the one hand, grooves are generally considered to be formed on the top of ripples, which is confirmed by our measurements with one-beam irradiation (equivalent to zero delay $\Delta \mathrm{t}=0$ ). On the other hand, in two-beams irradiation experiments $(\Delta t \neq 0)$, the grooves appeared on a flat surface, in absence of any sign of ripples, as evidence SEM images in Fig. 4 (e-f). Interestingly, grooves begin to appear at the time $\Delta t>\tau^{*}$, when LSFL vanishes. Since the formations of ripples and grooves at zero delay are connected (Fig. 4a-c), transient state X1 activated with $\mathrm{F}_{1}$ on the $\mathrm{X} 1 \leftarrow \mathrm{X} 0$ transition participates in excitation of a higher lying transient state $\mathrm{X} 2$, which results in the grooves formation (Fig. 9b). We assume that the activation energy density $\mathrm{E}_{a}$, grooves of $\mathrm{X} 2$ decreased along the reaction coordinate $\mathrm{Q}$ of $\mathrm{X} 1$ towards the final surface morphology. If this decrease is stronger than energy losses of $\mathrm{X} 1$ state, $\mathrm{X} 2 \leftarrow \mathrm{X} 0$ transition becomes accessible with fluence $\mathrm{F}_{2}$ at the critical time $\Delta \mathrm{t}=\tau_{\mathrm{g}}$ after the first beam absorption. Consequently, an increase of the laser energy would result in a shortening of the critical temporal delay between two laser beams permitting to reach $\mathrm{X} 2$ state, which is in agreement with Fig. 8 (b, f).
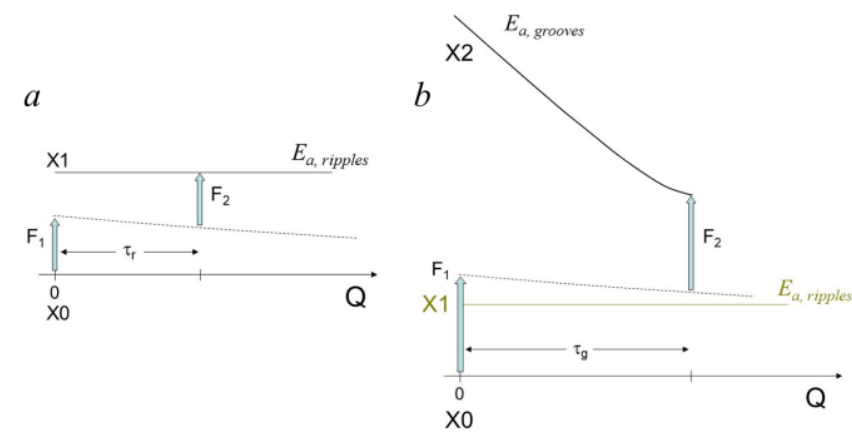

Fig. 9. Phenomenological schemas of structuring process in pumppump experiments. $F_{1}$ and $F_{2}$ are laser fluences of two spatially superposed beams and temporarily delayed by $\tau, \mathrm{Q}$ is reaction coordinate of the solid modification towards formation of NCS (a) and LSFL (b).

The asymmetry of FFA and grooves areas with respect to the temporal delay (positive $\Delta t>0$ or negative $\Delta t<0$ ) between two laser beams with different energies $\mathrm{E}_{1} \neq \mathrm{E}_{2}$ supports the proposed schema in Fig. 9b. In our pump-pump experiments with disproportioning energies of two laser beams, the reference beam was that with a weaker fluence $\mathrm{F}_{1}$, while the stronger one with fluence $F_{2}>F_{1}$ was sent before $(\Delta t<0)$ and after $(\Delta t>0)$ the reference beam. Because of the excited state energy depletion, the electronic energy density stored in the final state is $\mathrm{E}(\tau)=$ $E_{i} \cdot e^{-k}\left|\Delta_{t}\right|+E_{j}(i \neq j=1$ or 2$)$ and is stronger when $\mathrm{i}=1$ and $\mathrm{j}=2$, which facilitates attaining the X2 state potential. This is in agreement with the experimental results reported in Figs 6a (grooves) and $6 \mathrm{~b}$ (FFA), where critical time of the structures appearance is shorter for the positive delays $\Delta t>0$, as compared with $\Delta t<0$.

The proposed schema of the surface structures activation by lasers fits that proposed for controlling the reaction selectivity via the propagation time on the excited state potential energy surface, by applying delayed femtosecong pulses [7]. The case of grooves / FFA morphologies can be treated by considering the total energy evolution in the crystal. The interaction of light with an excited surface at intermediate reaction times can cancel LSFL (ripples), while maintain them at zero and infinite delays after the process beginning. Furthermore, an appropriately chosen delay time between femtosecond pulses permits to significantly decrease energy required for the surface modification: in case of FFA and grooves the energetic efficiency of the structuring process was increased by a factor of 3 .

A theoretical study of physical processes triggered by HDEE regime in rutile $\mathrm{TiO}_{2}$ crystals with (100) and (001) surface orientations has been recently reported [30]. This work has shown a strong enhancement of the absorption of the second laser pulse (delayed by $\Delta \mathrm{t}$ ) in the lattice modified with the first pulse, creating necessary conditions for a significant weakening of onset energies for the grooves and FFA formation. In framework of the proposed schema, these energy states characterize the structure of superheated material and transient liquid phase, which remains largely unknown. These material states depend on the electronic excitation and lattice temperature, and the energy is a complex function of the charge carrier density $\mathrm{N}_{\mathrm{e}}$ and interatomic distances $\mathrm{r}$, which depend on the lattice temperature $\mathrm{T}_{\mathrm{L}}$ of the solid. The relevant transient states are by consequence complex surfaces of energy density E(r, $\mathrm{N}_{\mathrm{e}}$ ). A view of the electronic transitions in a frame $\left[\mathrm{E}, \mathrm{r}, \mathrm{Ne}_{\mathrm{e}}\right]$ leading to the formation of grooves is given in Fig. 10.

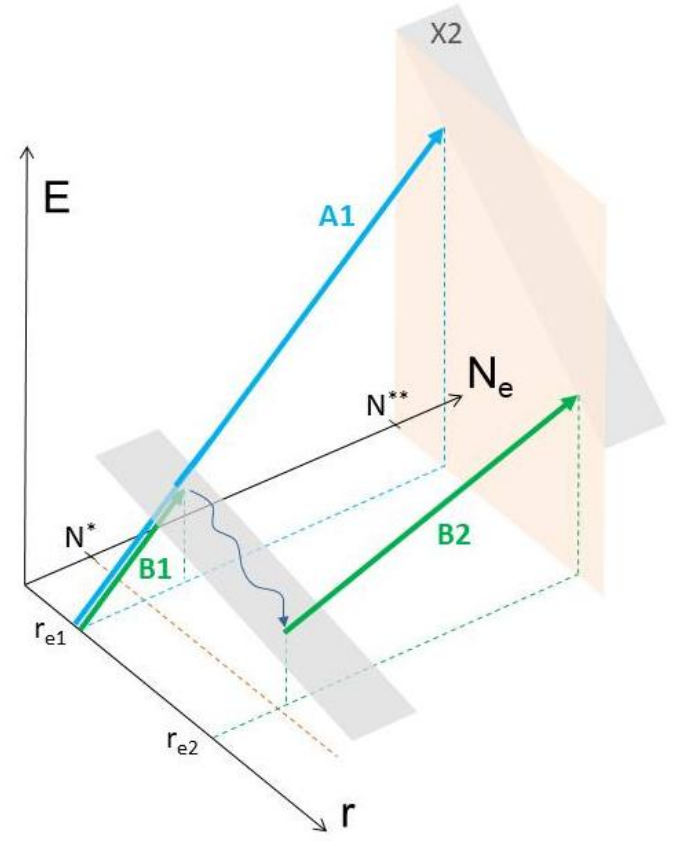

Fig. 10. View of electronic transitions in frame $\left(\mathrm{E}, \mathrm{r}, \mathrm{N}_{\mathrm{e}}\right)$ leading to formation of grooves (see explanation in text). Laser fluence in one-beam is $F_{A 1}$ and laser fluences in two-beams are $F_{B 1}$ and $F_{B 2}$, $\mathrm{N}_{\mathrm{e}}$ is charge carrier density and $\mathrm{r}$ is characteristic interatomic distance. 
The critical charge density $\mathrm{N}^{* *}$ for the formation of grooves can be attained by applying one laser pulse $\mathrm{A} 1$ to $\mathrm{TiO}_{2}(\mathrm{~T}=300 \mathrm{~K}$, $\left.\mathrm{r}=\mathrm{r}_{\mathrm{e} 1}\right)$ : this corresponds to the vertical electronic transition in $(\mathrm{E}$, r) plane and inclined transition in $\left(\mathrm{E}, \mathrm{N}_{\mathrm{e}}\right)$ plane. On the other hand, by applying first laser beam $\mathrm{B} 1\left(\mathrm{~T}_{\mathrm{L}}=300 \mathrm{~K}, \mathrm{r}=\mathrm{r}_{\mathrm{e} 1}\right)$ and second delayed B2 (after solid heating $\mathrm{T}_{\mathrm{L}} \gg 300 \mathrm{~K}: \mathrm{r}_{\mathrm{e} 1} \rightarrow \mathrm{r}_{\mathrm{e} 2}$ ) $\mathrm{N}^{* *}$ can be attained at a smaller energy cost: $\mathrm{F}_{\mathrm{B} 1}+\mathrm{F}_{\mathrm{B} 2}<\mathrm{F}_{\mathrm{A} 1}$. In this regard, a contribution of the electronically melt state to the critical delay $\tau_{\mathrm{r}}$ may take place, as it has been previously reported in GaAs [11]. On longer times, the hypothetical state X2 can result in thermal gradients within the fluid melt characterized by large Prandtl numbers [37].

Despite of the tentative explanation has been given in Ref. [30], the qualitative aggreement between theory and experiment using delayed laser beams is not yet attained. In particular, relatively narrow delay time-window for the initiation of suprawavelength periodic surface structures and amplification/annihilation of LSFL with the temporal delay for $\mathrm{E}_{1,2}>\mathrm{E}^{*}$ remain unexplored. In these conditions, theoretical picture of the transient electronic states evolution on the picosecond timescale needs a better understanding.

\section{Conclusions}

In this study, we report on the application of delayed femtosecond laser beams to control the final surface morphology of solids in the regime of high density electronic excitation. Single rutile $\mathrm{TiO}_{2}$ crystals with (001) and (100) surface orientations were irradiated with repetitive pulses of a femtosecond $\mathrm{KrF}$ laser (450 fs, $248 \mathrm{~nm}$ ), which photon energy is higher than that of the interband transitions in solid: $h v>\mathrm{E}_{\mathrm{g}}=3.0 \mathrm{eV}$ This excitation created a huge number density of the electron-hole plasma that induced surface modifications resulting in nanoparticle-covered structures (NCS), low-spatial frequency LIPSS (LSFL), grooves and featureless flat area (FFA). The excitation beams quality (Gaussian), fluence and superposition on target were permanently monitored in these experiments.

The interaction of light with transient surface states can lead to the onset and suppression of LSFL, grooves and FFA structures, providing physical control of the surface modification. The process deactivation time was estimated to be $\tau^{*}=6 \pm 1$ ps close to the threshold fluence. A strong decrease by a factor of 3 of the grooves onset energy was observed on the (001) oriented crystal after introduction of a temporal delay of $\sim 8 \mathrm{ps}$ between two laser beams. The last effect was confirmed on the (100) oriented crystal, however unusual FFA was observed instead of grooves, which is connected to the crystal anisotropy. The mechanism of the grooves and FFA formation in experiments with two delayed laser beams are different from that triggered by one laser pulse. We concluded that the both structures are promoted by the same sequence of transient energy states; this conclusion is supported by the analysis of variation of the critical temporal delay with laser fluence, required for the disappearance of LSFL and appearance of grooves and FFA. The experimental observations were rationalized in the framework of a phenomenological model describing evolution of transient states energy along the reaction coordinate.

As we stated, the critical delays between two pulses $\tau_{\mathrm{r}}$ and $\tau_{\mathrm{g}}$ separate two domains of ripples $\left(\mathrm{t} \leq \tau_{\mathrm{r}}\right)$ and grooves/FFA $\left(\mathrm{t} \geq \tau_{\mathrm{g}}\right)$ formation. We notice that one broad pulse of the shorter duration is expected to trig ripples. In contrast, by using one longer pulse the process coherence will be disturbed, which would result in a poor-resolved picture of the surface structuring. Furthermore, recent theoretical modelling [30] predict in this case a loss of efficiency of the grooves/FFA formation.

\section{Acknowledgments}

This work was carried out in part at the Ultraviolet Laser Facility operating at IESL-FORTH (no. 002258) with the financial support from the European Union's Horizon 2020 research and innovation program under grant agreement no 654148; G.D.T acknowledges financial support from COST Action TUMIEE (supported by COST-European Cooperation in Science and Technology). The authors are indebted to Apostolis Englezis for invaluable technical support.

\section{References}

1. A.H. Zewail, Femtochemistry, J. Phys. Chem. 97 (1993) 1242112446.

2. A.H. Zewail, "Femtochemistry: Atomic-Scale Dynamics of the Chemical Bond Using Ultrafast Lasers" (Nobel Lecture), Angew. Chem. Int. Ed. Engl. 39 (2000) 2586-2631.

3. T. Amand, V. Blanchet, B. Girard, X. Marie, Coherent Control in Atoms, Molecules and Solids, in: C. Rullière (ed.) Femtosecond Laser Pulses, Advanced Texts in Physics, Springer, NY, 2005.

4. H. Katsuki, N. Takei, C. Sommer, K. Ohmori, Acc. Chem. Res. 51 (2018) 1174-1184.

5. D.A. Cherepanov, F.E. Gostev, I.V. Shelaev, N.N. Denisov, V.A. Nadtochenko, Monitoring the Electric Field in CdSe Quantum Dots Under Ultrafast Interfacial Electron Transfer via Coherent Phonon Dynamics, Nanoscale 10 (2018) 22409-22419.

6. J.G. Horstmann, H. Böckmann, B. Wit, F. Kurtz, G. Storeck, C. Ropers, Coherent control of a surface structural phase transition, Nature 583 (2020) 232-236.

7. D.J. Tannor, R. Kosloff, S.A. Rice, Coherent pulse sequence induced control of selectivity of reactions: Exact quantum mechanical calculations, J. Chem. Phys. 85 (1986) 5805-5820.

8. N. Itoh, A.M. Stoneham, Treatment of semiconductor surfaces by laser-induced electronic excitation, J. Phys.: Condens. Matter 13 (2001) R489-R503.

9. N.F. Mott, Metal-Insulator Transitions, Taylor \& Francis, London, 1974.

10. S.K. Sundaram, E. Masur, Inducing and probing nonthermal transitions in semiconductors using femtosecond laser pulses, Nat. Mater. 1 (2002) 217-224.

11. C.W. Siders, A. Cavalleri, K. Sokolowski-Tinten, C. Toth, T. Guo, M. Kammler, M.H. von Hoegen, K.R. Wilson, D. von der Linde, C.P. Barty, Detection of nonthermal melting by ultrafast X-ray diffraction, Science 286 (1999) 1340-1342.

12. A. Rousse, C. Rischel, S. Fourmaux, I. Uschmann, S. Sebban, G. Grillon, Ph. Balcou, E. Förster, J. P. Geindre, P. Audebert, J.C. Gauthier, D. Hulin, Nonthermal melting in semiconductors measured at femtosecond resolution, Nature 410 (2001) 65-68.

13. J.S. Graves, R.E. Allen, Response of GaAs to fast intense laser pulses, Phys. Rev. B 58 (1998) 1362713633. 
14. A.Y. Vorobyev, C. Guo, Direct femtosecond laser surface nano/microstructuring and its applications, Laser \& Photonics Rev. 7 (2012) 385-407.

15. E. Stratakis, Nanomaterials by Ultrafast Laser Processing of Surfaces, Sci. Adv. Mater. 4 (2012) 407431.

16. J. Bonse, J. Krüger, S. Höhm, A. Rosenfeld, Femtosecond laser-induced periodic surface structures, J. Laser Appl. 24 (2012) 042006.

17. A. Papadopoulos, E. Skoulas, G.D. Tsibidis, E. Stratakis, Formation of periodic surface structures on dielectrics after irradiation with laser beams of spatially variant polarisation: a comparative study, Appl. Phys. A 124 (2018) 146.

18. K.C. Phillips, H.H. Gandhi, E. Mazur, S.K. Sundaram, Ultrafast laser processing of materials: a review, Adv. Opt. Photonics 7 (2015) 684-712.

19. J. Bonse, S. Höhm, S.V. Kirner, A. Rosenfeld, J. Krüger, Laser-induced periodic surface structures: A scientific evergreen, IEEE J. Sel. Top. Quant. 23 (2017) 9000615 .

20. G.D. Tsibidis, C. Fotakis, E. Stratakis, From ripples to spikes: A hydrodynamical mechanism to interpret femtosecond laser-induced self-assembled structures, Phys. Rev. B 92 (2015) 041405(R).

21. S. He, J.J.J. Nivas, K.K. Anoop, A. Vecchione, M. Hu, R. Bruzzese, S. Amoruso, Surface structures induced by ultrashort laser pulses: Formation mechanisms of ripples and grooves, Appl. Surf. Sci. 353 (2015) 12141222 .

22. E. Stratakis, J. Bonse, J. Heitz, J. Siegel, G. D. Tsibidis, E. Skoulas, A. Papadopoulos, A. Mimidis, J. Commans, J. Kruger, C. Florian, Y. Fuentes-Edfuf, J. Solis, W. Baumgartner, Laser engineering of biomimetic surfaces, Mater. Sci. Eng., R141 (2020) 100562

23. A. Y. Vorobyev, C. Guo, Direct femtosecond laser surface nano/microstructuring and its applications, Laser Photonics Rev. 7 (2013) 385-407.

24. J. Bonse, S. Gräf, Maxwell Meets Marangoni - A review of theories on laser-induced periodic surface structures, Laser Photonics Rev. 14 (2020) 2000215.

25. J. Reif, Material Response to Laser Energy Deposition (Thermal and Hyperthermal Processes), in: M. Castillejo, P.M. Ossi L. Zhigilei (eds) Lasers in Materials Science, Springer, NY, 2014.

26. J. Reif, F. Costache, S. Eckert, The role of energy and phase relaxation ( $\mathrm{T} 1$ and $\mathrm{T} 2$ ) in ultrafast laser ablation, J. Phys. Conf. Ser. 59 (2007) 1-4.

27. V. Schmidt, W. Husinsky, G. Betz, Dynamics of laser desorption and ablation of metals at the threshold on the femtosecond time scale, Phys. Rev. Lett. 85, (2000) 3516-3519.

28 J. Reif, O. Varlamova, M. Bounhalli, M. Muth, T. Arguirov, "Nanostructure formation upon femtosecond ablation from silicon: Effect of double pulses." Appl. Surf. Sci. 258 (2012) 9491-9495.

29. L. Museur, G.D. Tsibidis, A. Manousaki, D. Anglos, A. Kanaev, Surface structuring of rutile $\mathrm{TiO}_{2}$ (100) and (001) single crystals with femtosecond pulsed laser irradiation, J. Opt. Soc. Am. B 35 (2018) 2600-2607.

30. G.D. Tsibidis, L. Museur, A. Kanaev, Role of crystalline orientation in the formation of surface patterns on solid irradiated with femtosecond laser double pulses, Appl. Sci. 10 (2020) 8811.

31. D. Reyes-Coronado, G. Rodriguez-Gattorno, M.E. Espinosa-Pesqueira, C. Cab, R. de Coss, G. Oskam, Phase-pure $\mathrm{TiO}_{2}$ nanoparticles: anatase, brookite and rutile, Nanotechnol. 19 (2008) 145605.

32. J.E. Sipe, J.F. Young, J.S. Preston, H.M. van Driel, Laser-induced periodic surface structure. I. Theory, Physical Review B 27 (1983) 1141-1154.

33. A. Margiolakis, G.D. Tsibidis, K.M. Dani, G.P. Tsironis, Ultrafast dynamics and subwavelength periodic structure formation following irradiation of GaAs with femtosecond laser pulses, Phys. Rev. B 98 (2018) 224103.

34. B. Gaković, G.D. Tsibidis, E. Skoulas, S.M. Petrović, B. Vasić, E. Stratakis, Partial ablation of Ti/Al nano-layer thin film by single femtosecond laser pulse, J. Appl. Phys. 122 (2017) 223106.

35. G.D. Tsibidis, E. Skoulas, E. Stratakis, Ripple formation on nickel irradiated with radially polarized femtosecond beams, Opt. Lett. 40 (2015) 5172-5275.

36. G.D. Tsibidis, M. Barberoglou, P.A. Loukakos, E. Stratakis, C. Fotakis, Dynamics of ripple formation on silicon surfaces by ultrashort laser pulses in subablation conditions, Phys. Rev. B 86 (2012) 115316.

37. G.D. Tsibidis, E. Skoulas, A. Papadopoulos, E. Stratakis, Convection roll-driven generation of suprawavelength periodic surface structures on dielectrics upon irradiation with femtosecond pulsed lasers, Phys. Rev. B 94 (2016) 081305(R). 